\title{
Imbibition of conductive polymer ink on the nanopapers consisting of cellulose nanofibers fabricated from different protocols
}

Shingo Ohira ${ }^{1}$, Daiki Mizumura ${ }^{1}$, and Itsuo Hanasaki ${ }^{1 *}$

${ }^{1}$ Institute of Engineering, Tokyo University of Agriculture and Technology, Naka-cho 2-24-16, Koganei, Tokyo 184-8588, Japan

*E-mail: hanasaki@cc.tuat.ac.jp

Introduction. Nanopapers fabricated from cellulose nanofibers (CNFs) are expected as substrates of the printed electronics ${ }^{(1)}$. Nanopapers are not only environmentally friendly but also advantageous for drawing conductive wires on them because of the fine textures ${ }^{(2)}$. However, the CNFs are actually diverse in their specification originating from the production process, which might cause the difference in the inkdrawing characteristics of nanopapers. Therefore, we have examined the behaviors of droplets of conductive ink on nanopapers fabricated from different CNFs.

Experimental method. Drying the aqueous dispersions at initial concentration of $0.18 \mathrm{wt} \%$ on slide glasses with side walls of silicone sheets, two types of nanopapers were fabricated from different CNFs. One type of CNFs was mehcanically defibrated and the other was TEMPOoxidized. The dispersions had been evacuated before drying, and the drying temperature was $30 \mathrm{deg}$.C on a hotplate $^{(3)}$. The unit dispersion amount was $3739 \mu \mathrm{L}$ on the hole with a bottom area of $53 \mathrm{~mm} \times 20 \mathrm{~mm}$, and this drying process was repeated three times to attain a desired thickness of the nanopapers. Then, we examined the PEDOT:PSS ink droplet behaviors on these nanopapers by inkjet discharge on a spot for specified repetitions. The droplet images were captured from side and top views to examine the imbibition characteristics.

Results and discussion. Fig. 1 shows the time evolution of PEDOT:PSS ink droplets on the two types of nanopapers. While the inkjet discharge is the same condition, the droplet shapes are significantly different when the constituent CNFs are different. The nanopaper made of mechanically defibrated CNFs shows more wettability at the dimensionless time $t^{*}=0$ and 0.5 , where the time is scaled by the drying duration. Fig. 2 shows the experimentally measured radii of ink droplets based on different view angles. The radii of droplets on nanopapers from mechanically defibrated CNFs are larger than those from TEMPO-oxidized CNFs, which is also qualitatively recognized from Fig.1. Furthermore, the former shows even larger size from top view compared to those from side view, whereas the latter shows rather slightly smaller size from the top view. Larger radius from top view is caused by the penetration of the ink into the nanopapers. Thus, the PEDOT:PSS ink on the nanopaper made from the mechanically defibrated CNFs penetrated into the nanopapers more than those from the TEMPO-oxidized CNFs. This is at least partly related to the difference of wettability as shown in Fig.1.

Conclusion. We examined the imbibition behaviors of conductive polymer ink droplets on nanopapers fabricated from different types of CNFs. Within the range investigated here, the nanopapers made of TEMPO-oxidized CNFs keeps more ink on the surface with less imbibition compared to the nanopapers made of mechanically defibrated CNFs.

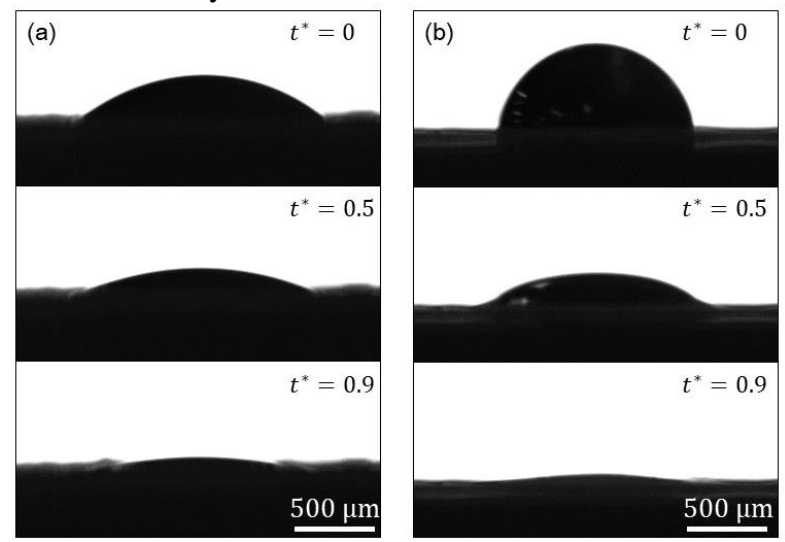

Fig.1 Time evolution of the 200-shot droplets of conductive polymer ink on the nanopapers fabricated from (a) mechanically defibrated and (b) TEMPOoxidized CNFs.

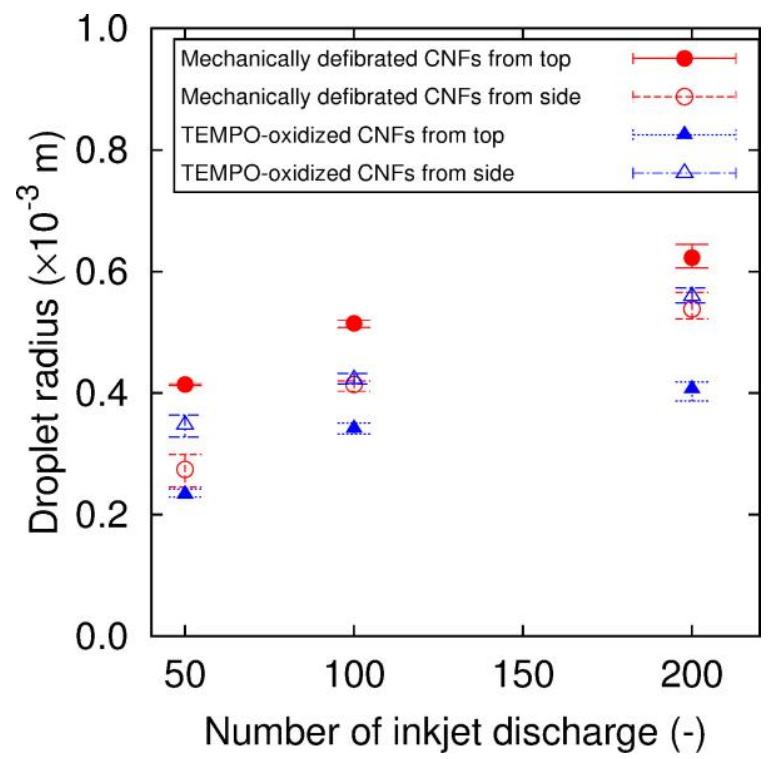

Fig. 2 Droplet radius measured from side and top views. The side view is based on the maximum value during the whole drying process. The error bars indicate the maximum and minimum values among the three trials.

\section{References.}

(1) Hoeng, F., Denneulin, A., and Bras, J., Nanoscale, 8, (2016), pp. 13131-13154.

(2) Hsieh, M., Kim, C., Nogi, M., and Suganuma, K., Nanoscale, 5, (2013), pp. 9289-9295.

(3) Mizumura, D., Hanasaki, I., Ooi, Y., Horikawa., Y., Micro \& Nano Lett., 12, (2017), pp. 516-519. 